but 3 out of 4 DOT1L inhibited samples showed a significantly reduced effect of TGF $\beta$ on ACTA2 expression. The amount of collagen I and III in the extracellular matrix after 72 hours of TGF $\beta$, was comparable between control and EPZ treated fibroblasts. BrdU labelling assay showed increased proliferation with DOT1L inhibition. In vivo, subcutaneous bleomycin induced an increased dermal thickness and skin collagen content in mice. No difference in the effect of bleomycin was found between mice with a conditional fibroblastspecific DOT1L knockout or wild type mice.

Conclusions In an in vitro model of fibrosis, primary human dermal fibroblasts treated with a DOT1L-inhibitor showed increased proliferation and reduced upregulation of ACTA2 but did not result in detectable differences in collagen deposition. In an in vivo murine model of skin fibrosis, no difference in bleomycin-induced skin thickness and collagen content was found when DOT1L was knocked out in fibroblasts.

Disclosure of interest None declared

\section{P102 S100A9 HAMPERS OSTEOCLAST DIFFERENTIATION FROM CIRCULATING PRECURSORS BY REDUCING THE EXPRESSION OF RANK}

${ }^{1} \mathrm{M}$ van den Bosch, ${ }^{1} \mathrm{I}$ Di Ceglie, ${ }^{2} \mathrm{~T}$ Vogl, ${ }^{2} \mathrm{~J}$ Roth, ${ }^{3} \mathrm{C}$ Goodyear, ${ }^{1} \mathrm{P}$ van der Kraan, ${ }^{1} \mathrm{~A}$ Blom, ${ }^{1} \mathrm{P}$ van Lent*. ${ }^{1}$ Radboud university medical centre, Nijmegen, Netherlands; ${ }^{2}$ University of Muenster, Muenster, Germany; ${ }^{3}$ University of Glasgow, Glasgow, UK

\subsection{6/annrheumdis-2018-EWRR2018.118}

Introduction The alarmins S100A8/A9 are produced in high levels in the synovium during both experimental and human rheumatoid arthritis (RA) and have been implicated in inflammation-induced bone resorption. We and others have previously shown that stimulation of mature osteoclasts with S100A8/A9 results in increased numbers and resorptive activity. In agreement, reduced bone destruction was observed after induction of experimental RA models in S100a $9^{-/-}$mice. However, the effects of S100A8/A9 on monocyte-to-osteoclast differentiation remain elusive.

Objectives Here, we investigated the effects of S100A9 on CD $14^{+}$monocytes and their potential to differentiate into osteoclasts.

Methods CD14 ${ }^{+}$monocytes were isolated from buffy coats of healthy donors using density gradient centrifugation and magnetic cell sorting. Cells were differentiated into osteoclasts with macrophage colony-stimulating factor (M-CSF) and Receptor activator of nuclear factor kappa-B (RANK) ligand (RANKL) in the presence or absence of S100A9. mRNA expression was determined by RT-qPCR and protein expression was determined using Luminex analysis. Moreover, osteoclast differentiation was assessed using Tartrate-resistant acid phosphatase (TRAP) staining and the resorptive capacity was determined using mineral-coated plates. RANK protein expression was assessed using FACS.

Results We observed that S100A9 stimulation of monocytes resulted in a strong induction of various pro-inflammatory factors, such as interleukin (IL)1 $\beta$, IL6, IL8, and tumour necrosis factor (TNF) $\alpha$ after 24 hour, both on the mRNA and protein level. Interestingly, we observed a strong decrease in the number of multinucleated osteoclasts as determined by TRAP staining, at day 6 and 8 after start of the cultures. In agreement with this, the cells showed a strongly reduced resorptive capacity. We demonstrated that already a 24 hour stimulation with S100A9 strongly reduced the osteoclastogenic potential of the $\mathrm{CD}^{+} 4^{+}$monocytes. Finally, we observed that S100A9 stimulation hampered the M-CSF-induced upregulation of RANK, which could be reversed by addition of the TNF $\alpha$ inhibitor etanercept, but not the interleukin 1 receptor antagonist.

Conclusions Whereas S100A8/A9 have been previously shown to stimulate the numbers and resorptive capacity of mature osteoclasts, we here show that stimulation of monocytes with S100A9 strongly inhibits their osteoclastogenic potential, possibly via $T N F \alpha$-induced reduction of RANK expression. This suggests that the timing of exposure to S100A8/A9 is an important determinant for monocyte-to-osteoclast differentiation.

Disclosure of interest None declared

\section{P103 PRO-FIBROTIC RESPONSES INDUCED BY THYMIC STROMAL LYMPHOPOETIN}

${ }^{1} \mathrm{~L}$ Wang, ${ }^{2} \mathrm{~S}$ Wang, ${ }^{3} \mathrm{~V}$ Taneja, ${ }^{2} \mathrm{R}$ Vassallo. 'Department of Pulmonary Medicine, Guang'anmen Hospital, China Academy of Chinese Medicine Science, Beijing, China; ${ }^{2}$ Pulmonary and Critical Care Medicine; ${ }^{3}$ Immunology, Mayo Clinic, Rochester, Minnesota, United States

\subsection{6/annrheumdis-2018-EWRR2018.119}

Registration status I still need to register to EWRR Introduction Thymic Stromal Lymphopoetin (TSLP) is a T helper-2 (Th2) cytokine that may be an important regulator of tissue fibrosis. ${ }^{1}$ Rheumatoid Arthritis (RA)-associated interstitial lung disease is a fibrotic extra-articular complication associated with dysregulated adaptive immunity in the lung. In a recent study - Vassallo et al. Clin Immunol 2014;152(1-2):25-35 we reported increased TSLP mRNA levels in the lungs of cigarette smoke-exposed, collagen induced arthritis mice with a DQ8 background. ${ }^{2}$

Objectives The purpose of this study was to explore direct molecular mechanisms by which TSLP induces fibrogenesis.

Methods We performed in vitro studies with human lung fibroblasts (Lonza) incubated with recombinant human TSLP (R\&D Systems, 0-10 ng/ml) and determined TSLP-induced proliferation using a CyQuant assay, as well as collagen and fibronectin gene expression. We also investigated signaling intermediates activated by TSLP utilizing a Signal Transduction and Activator of Transcription (STAT)1-6 ELISA assay on lysates generated from TSLP stimulated fibroblasts at varying time points.

Results Human lung fibroblasts proliferate directly in response to TSLP with concentrations $\geq 1 \mathrm{ng} / \mathrm{ml}$ inducing a significant proliferative response compared with controls. TSLP also induced fibronectin and collagen gene expression in lung fibroblasts. Incubation with TSLP induced phosphorylation of STAT-1 and STAT-3: phosphorylation of STAT-2, as well as 4-6 was not observed. Phosphorylation of STAT-1 and 3 were observed within 30 minutes of incubation with TSLP (10 ng/ $\mathrm{ml})$.

Conclusions These in vitro studies demonstrate that fibroblasts are directly responsive to recombinant human TSLP which induces proliferation and matrix generation, potentially through activation of STAT-1 and 3. These observations further support a role for TSLP as a regulator of tissue fibrosis in the lung, and suggest a potential therapeutic target for the treatment of autoimmunity related fibrosis. 